\title{
Effects of Gene Imbalance on Amyloid Precursor Protein [APP] Expression in Grafts and Recipient Cortex
}

\author{
C.F. Hohmann ${ }^{1,2}$, G.T. Capone ${ }^{1}$, J.T. Coyle ${ }^{3}$ \\ The Kennedy Krieger Institute ${ }^{1}$ and Department of Psychiatry ${ }^{2}$, Johns Hopkins University, Baltimore, MD \\ and the Department of Psychiatry ${ }^{3}$, Harvard University, Boston, MA, USA
}

Individuals with trisomy, most prominently among them, Down's Syndrome, present with abnormal brain development and mental retardation. Whether such abnormal brain development is the consequence of enhanced levels of expression of all triplicated genes or the result of altered expression of certain developmentally important genes has long been unclear. Trisomy of mouse chromosome 16[Ts16] shares phenotypic and genotypic similarities with human trisomy 21, Down's Syndrome [DS], thus providing a suitable model to address the mechanism by which gene imbalance may affect brain ontogeny. However, Ts16 does not survive past late gestation. We chose transplantation of late embryonic Ts16 cortex into neonatal euploid hosts as a paradigm to examine later stages of forebrain development. Previous results with somatostatin and GAP-43, both located on chromosome 16, showed expression patterns that were specific to the transplant age and mostly differed from the host /2/. However, unlike Ts16 embryos, Ts16 grafts did not show elevated expression of genes located on chromosome 16 if compared to euploid, age matched grafts and/or host cortex. Thus, host and graft tissue appeared to interact with each other in regulating the expression of genes located on chromosome 16.

This study investigates the expression of APP peptides and mRNA in grafts of late embryonic Ts16 and euploid litter mate cortex transplanted to the neocortex of neonatal euploid hosts. At all ages, both Ts 16 and euploid grafts display some intensely APP immunoreactive [IR] cellular structures of unusual morphology near their borders with the host cortex. These may represent a glial response to transplantation "injury". Aside from these structures, APP-IR is compa- rable in both pattern and intensity in both Ts 16 and euploid grafts as well as in host tissue at postnatal day [PND] 7 after transplantation. In contrast, by PND 14 a "halo" of diffuse IR is present in and around the grafts; this is particularly apparent in the Ts 16 grafts. This localized elevation in IR is transient and can no longer be observed in cases one month post-transplantation and older. However, many of the hosts carrying Ts 16 transplants begin to show a severely disrupted pattern of IR in the CA3 region of the hippocampus. Similar pathology was observed previously by us in host animals following the grafting of $\mathrm{PC} 12$ cells transformed with the carboxy-terminal 105 amino acids of APP. These APP-IR changes in the host hippocampus resemble the effects of intraventricular excitatory amino acid injections in the adult rodents $/ 1,5 /$, with the exception that adjacent Nissl stained sections did not show neuronal loss.

Expression of mRNA for APP, as analyzed by in situ hybridization, shows an age dependent pattern in both Ts16 and euploid graft that differs from the host cortex. As in the IR pattern, a transient elevation is observed in both Ts16 and euploid transplants at PND 14 with younger and older grafts exhibiting below host levels of mRNA expression. Expression is the lowest in aged grafts, 1 year after transplantation, for both Ts16 and euploid. Thus, unlike in the Ts16 embryos /1/, APP mRNA is not generally elevated above euploid levels in the Ts 16 grafts. However, preliminary results suggest differences in the time course and levels of APP mRNA expression in Ts16 vs. euploid grafts when expression is measured as percentage of host cortex.

The current results are compatible with the notion that APP is a developmentally regulated 


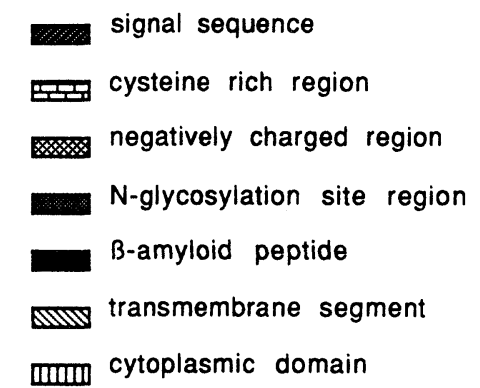

Reagents:

$\mathrm{F} 1$ and $\mathrm{B} 5$ antibodies showed comparable staining patterns in BALB/CByJ mice. F1 was used for the present study. The cDNA shown below was used for in situ hybridization experiments

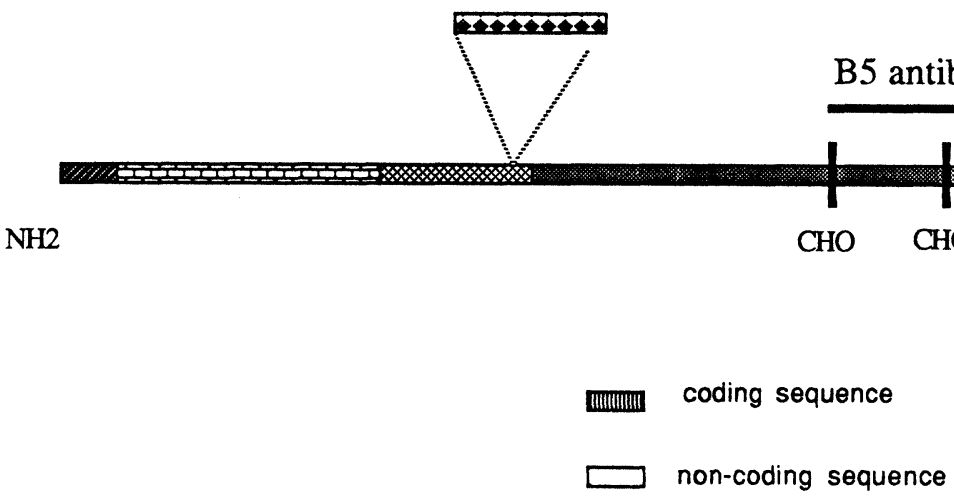

gene with a role in cortical morphogenesis. The data support previous results with transplants of PC12 cells expressing an APP peptide / $3 /$ which show that abnormal levels of APP peptide expression (C104) during development can lead to hippocampal pathologies as the animal matures. However, unlike some other investigators, we have failed to observe age dependent pathomorphology or amyloid formation directly associated with the Ts16 grafts or surrounding host cortex. Finally, as seen for SOM and GAP433, APP protein and mRNA expression appear to be regulated in the grafts in ways that differ from normal development but are not primarily a function of gene dosage. 

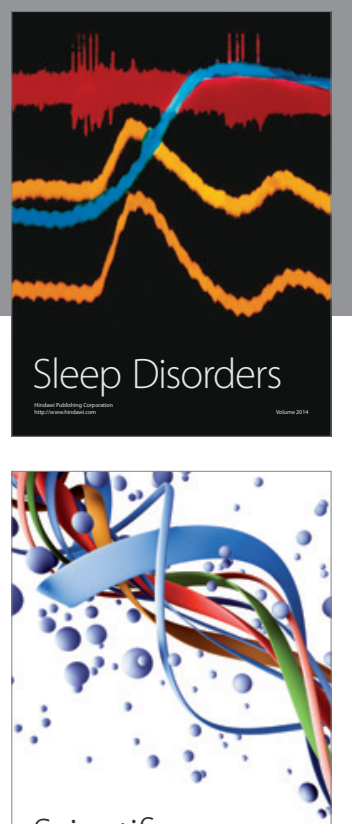

Scientifica
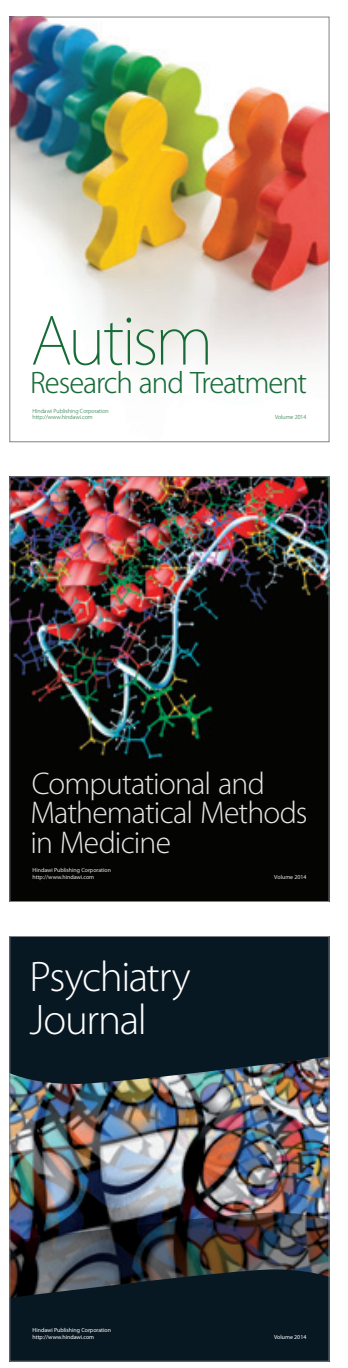
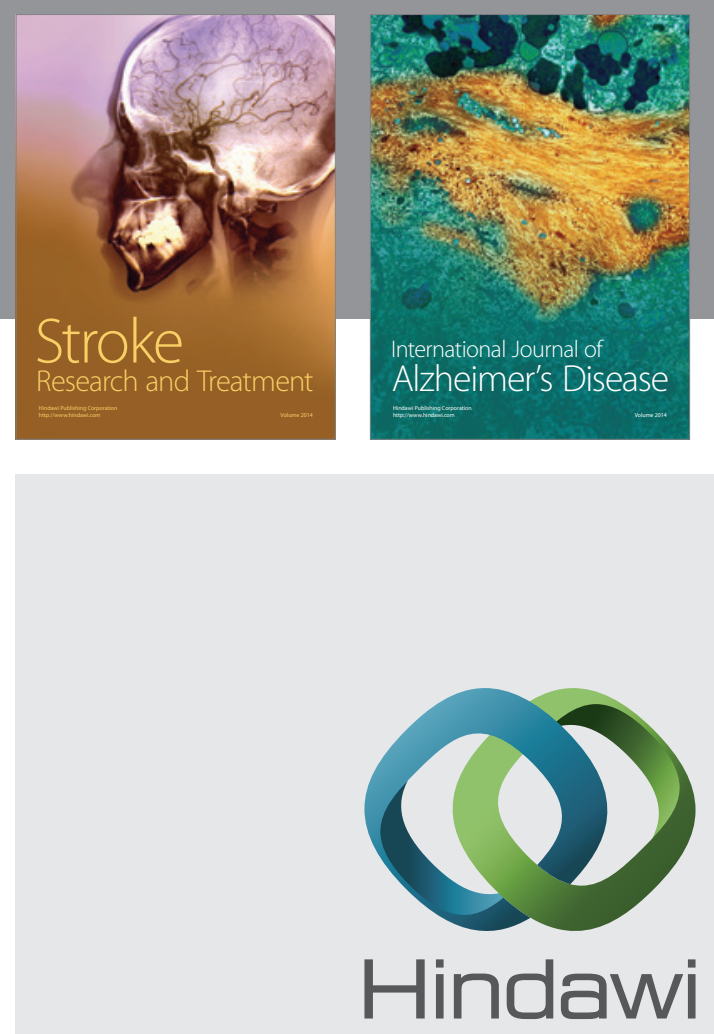

Submit your manuscripts at

http://www.hindawi.com
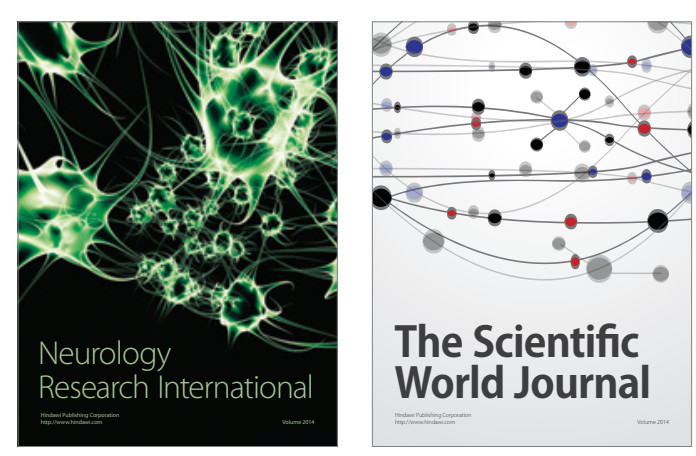

The Scientific World Journal

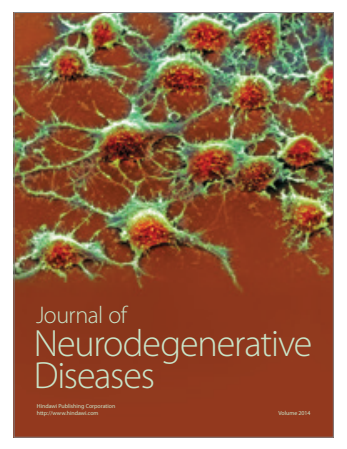

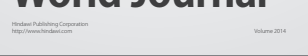

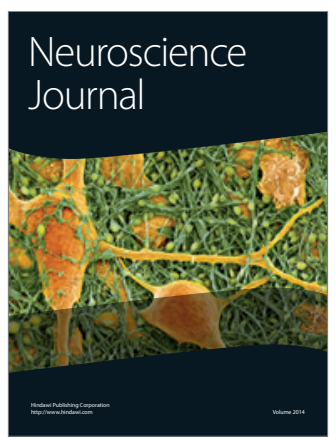

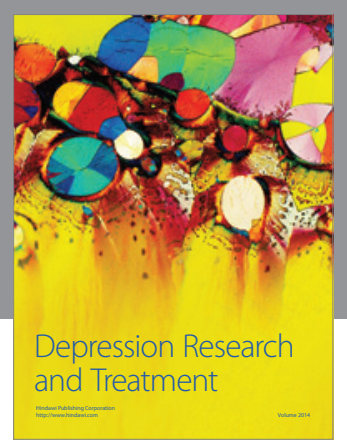
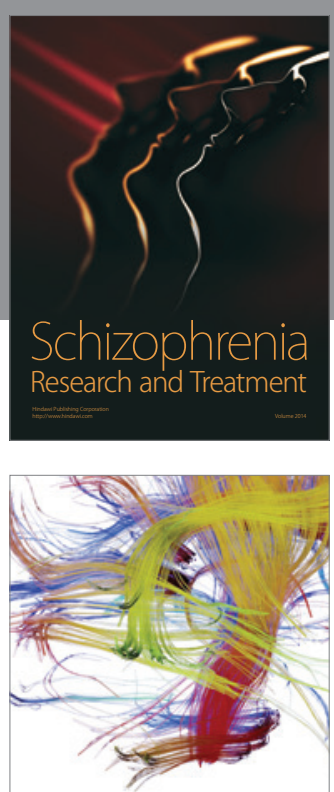

Brain Science

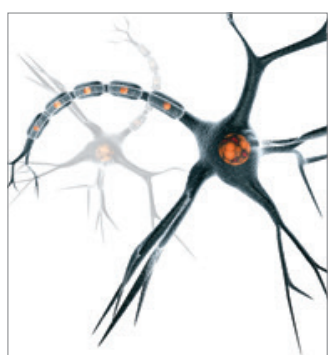

Neural Plasticity
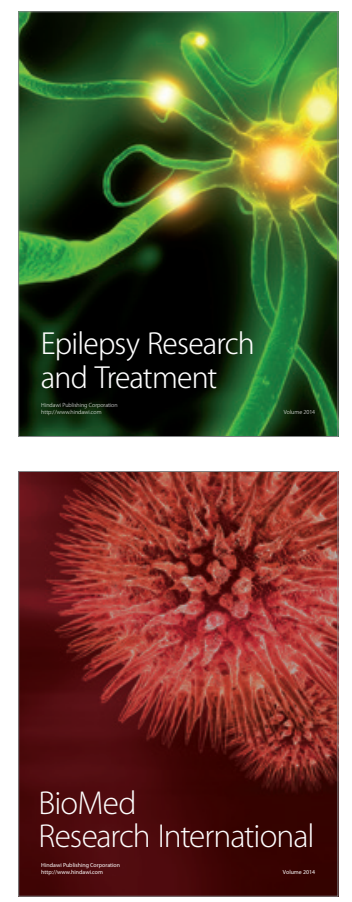

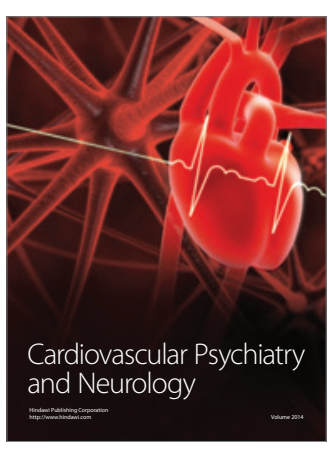

Parkinson's

Disease
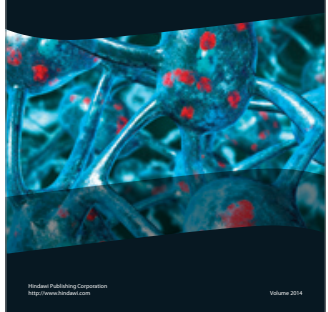